\title{
A Common Weight Linear Optimization Approach for Multicriteria ABC Inventory Classification
}

\author{
S. M. Hatefi ${ }^{1}$ and S. A. Torabi ${ }^{2}$ \\ ${ }^{1}$ Faculty of Engineering, Shahrekord University, Rahbar Boulevard, P.O. Box 115, Shahrekord 34141-88186, Iran \\ ${ }^{2}$ School of Industrial Engineering, College of Engineering, University of Tehran, Tehran 14174, Iran
}

Correspondence should be addressed to S. M. Hatefi; smhatefi@alumni.ut.ac.ir

Received 6 May 2014; Revised 22 October 2014; Accepted 11 December 2014

Academic Editor: Roger Z. Ríos-Mercado

Copyright (c) 2015 S. M. Hatefi and S. A. Torabi. This is an open access article distributed under the Creative Commons Attribution License, which permits unrestricted use, distribution, and reproduction in any medium, provided the original work is properly cited.

\begin{abstract}
Organizations typically employ the $\mathrm{ABC}$ inventory classification technique to have an efficient control on a huge amount of inventory items. The $\mathrm{ABC}$ inventory classification problem is classification of a large amount of items into three groups: A, very important; B, moderately important; and $\mathrm{C}$, relatively unimportant. The traditional $\mathrm{ABC}$ classification only accounts for one criterion, namely, the annual dollar usage of the items. But, there are other important criteria in real world which strongly affect the ABC classification. This paper proposes a novel methodology based on a common weight linear optimization model to solve the multiple criteria inventory classification problem. The proposed methodology enables the classification of inventory items via a set of common weights which is very essential in a fair classification. It has a remarkable computational saving when compared with the existing approaches and at the same time it needs no subjective information. Furthermore, it is easy enough to apply for managers. The proposed model is applied on an illustrative example and a case study taken from the literature. Both numerical results and qualitative comparisons with the existing methods reveal several merits of the proposed approach for $\mathrm{ABC}$ analysis.
\end{abstract}

\section{Introduction}

Inventory classification using $\mathrm{ABC}$ analysis, which is based on the Pareto principle, is one of the most widely employed inventory control techniques in practice $[1,2]$. ABC analysis classifies the inventory items or stock keeping units (SKUs) into three classes, namely, A (very important), B (moderately important), and C (relatively unimportant) requiring different level of control for each class and, at the same time, setting the same service level for all SKUs in a class. More details on inventory control policies for these classes of items can be found in Silver et al. [3].

Traditional $\mathrm{ABC}$ analysis considers only a single measure, most often annual dollar usage, to classify inventory items. However, in addition to this criterion, inventory holding unit cost, part criticality, length and variability of replenishment lead time, commonality, obsolescence, substitutability, number of requests per year, scarcity, durability, reparability, order size requirement, stockability, demand distribution, and stock-out unit penalty cost are recently recognized as other important criteria which affect inventory classification $[1,4,5]$. Thus, it can be realized that the traditional $A B C$ analysis may be an inefficient method for appropriate classification of inventory items in practice [6-8].

Several methods have been suggested to solve the problem of multiple criteria inventory classification (MCIC) in the literature. Bhattacharya et al. [9] and Rezaei and Dowlatshahi [10] provide comprehensive reviews of various methods introduced in the literature for MCIC issue. The implementation of analytic hierarchy process (AHP) to study this issue is addressed by Flores et al. [11] and Partovi and Burton [12]. More recently, a fuzzy AHP-DEA approach is proposed by Hadi-Vencheh and Mohamadghasemi [13] to solve the MCIC problem. However, when applying the AHP method, it is often a difficult task for the decision maker to accurately assign exact values to pairwise comparisons. Also, expert's opinion and judgment may play important role in deriving 
the criteria weights which consequently can affect $A B C$ classification results considerably.

Bhattacharya et al. [9] propose a distance-based multicriteria consensus framework based on the concepts of positiveideal and negative-ideal solutions for the $\mathrm{ABC}$ analysis. The authors also affirm that constructing fuzzy models such as fuzzy TOPSIS and neurofuzzy hybrid models would be suitable when considering the ambiguity of attribute values. An application of the case-based distance model to solve the MCIC problem can be seen in Chen et al. [14]. Recently, a new approach based on loss profit is proposed to deal with $\mathrm{ABC}$ analysis [15].

Application of artificial intelligence methods such as genetic algorithms, particle swarm optimization, and artificial neural networks to solve the MCIC problem can be found in Guvenir and Erel [6], Tsai and Yeh [16], and Partovi and Anandarajan [8], respectively. Artificial intelligence based classification techniques are addressed by $\mathrm{Yu}$ [17] to resort MCIC problem. Also, a fuzzy classification approach is proposed in the case where there exists nominal and nonnominal attributes [2]. Similarly, Rezaei and Dowlatshahi [10] present a rule-based method for classifying inventory items in a multicriteria setting. However, these methods are too complicated to be applied in practice so that they may not be easily understood by inventory managers.

A number of optimization based methods have also been developed to solve the MCIC problem. Ramanathan [1] proposes a weighted linear optimization model (hereafter Rmodel) for classifying inventory items with multiple criteria. The spirit of R-model is based upon the concept of data envelopment analysis (DEA) which has no subjectivity in determining the weights of criteria; that is, the weights are endogenously and repeatedly generated by a DEA-like model. Zhou and Fan [18] extend the R-model by incorporating some balancing features for MCIC using two sets of weights that are most favorable and least favorable for each item. Then, the final performance score of each item is obtained by aggregating the best and worst performance scores and using a control parameter called $\lambda$ whose value is determined by the decision maker subjectively. In a similar way, Chen [19] proposes the peer estimation approach in which the performance score of each inventory item is first measured in the most favorable and least favorable senses and then they are aggregated without any subjectivity. Chen [20] proposes another alternative model that resorts to virtual items and the concepts of TOPSIS for ABC analysis. The proposed model provides a unique inventory classification without any subjectivity.

Stanford and Martin [21] introduce a general ABC inventory classification system as the foundation for a normative model of the maintenance cost structure and stock turnover characteristics of a large, multi-item inventory system with constant demand. Ng [5] proposes a weighted linear optimization model for $\mathrm{ABC}$ inventory classification. The author also introduces a transformation technique to simplify the classification procedure (hereafter NG-model) which aids the inventory managers with obtaining the aggregated scores of inventory items without a linear optimizer. Hadi-Vencheh [22] provides an improved version of the NG-model which is a nonlinear programming model (hereafter HV-model). Both the NG and HV models require prior assumption on the importance order of the criteria which is subjectively determined by the decision maker. More recently, Teunter et al. [23] present an alternative approach for using multicriteria methodologies based on multiple way classifications. The authors propose a new cost criterion for ranking SKUs from an inventory cost perspective that accounts for demand volume, holding cost (purchase price), shortage cost (criticality), and average order quantity.

Park et al. [24] proposed a cross-evaluation-based weighted linear optimization model for the MCIC problem. Their proposed method incorporates a cross-efficiency evaluation approach into a weighted linear optimization model for finer classification of inventory items. For classification of inventory items, Soylu and Akyol [25] incorporated the preference of the decision-maker into the decision making process. They applied two utility-function-based sorting methods to solve the MCIC problem. Bacchetti et al. [26] proposed a hierarchical multicriteria classification method for inventory management purposes and applied it in a case study of the spare parts business of a household appliance manufacturer. Rezaei and Salimi [27] developed an interval programming model for $\mathrm{ABC}$ inventory classification. Their proposed model provides optimal results instead of an expert-based classification and it does not require precise values of item parameters. Torabi et al. [28] introduced an imprecise data envelopment analysis model to classify inventory items in the case where there exist both quantitative and qualitative criteria. Furthermore, Hatefi et al. [29] developed an iterative DEA-like model to solve the MCIC problem under quantitative and qualitative criteria. Lolli et al. [30] introduces a new hybrid method based on the AHP and the $\mathrm{K}$-means algorithm to solve the MCIC problem.

This paper proposes an alternative optimization-based model in which the composite performance scores of all inventory items are calculated simultaneously via a set of common weights. The proposed common weight linear optimization model has a notable computational saving in terms of the number of required LP models that must be solved and therefore can considerably reduce the processing time when controlling a large number of inventory items. Also, no subjective information is required to run the proposed model which is essential in an accurate and fair decision environment.

The rest of the paper is organized as follows. In Section 2, a brief discussion is provided about the R-model as the first optimization-based $\mathrm{ABC}$ classifier. Then, the proposed common weight linear optimization model is explained in detail. An application of the proposed model for $\mathrm{ABC}$ analysis is shown by a small numerical example as well as a case study taken from the literature in Sections 3 and 4 , respectively. Section 5 provides a comprehensive comparison and discussion about the proposed approach and the most relevant existing methods in the literature, that is, the R-model, the ZF-model, Chen's [20] model, the NG-model, and the HV-model. Finally, concluding remarks are reported in Section 6. 


\section{An Alternative Common Weights MCIC Approach}

Suppose that there are $M$ inventory items that are being classified as A, B, and C classes based on $N$ incommensurable criteria. Let $y_{m n}$ denote the value of criterion $n$ with respect to inventory item $m$. For simplicity, it is assumed that all the criteria are of benefit type, that is, positively related to the importance of an item. It is noteworthy to mention that the cost type criteria (i.e., those criteria that are negatively related to the importance level of items) can easily be converted into the benefit type ones by considering their reciprocal values [1]. Furthermore, in order to avoid any problem arising from the criteria scale differences, all measures are first normalized by using the following linear normalization procedure $[5,31]$ :

$$
\begin{array}{r}
\frac{y_{m n}-\min _{m=1,2, \ldots, M}\left\{y_{m n}\right\}}{\max _{m=1,2, \ldots, M}\left\{y_{m n}\right\}-\min _{m=1,2, \ldots, M}\left\{y_{m n}\right\}}, \\
j=1,2, \ldots, N .
\end{array}
$$

Notably, the above transformation formula converts all measurements into the $[0,1]$ interval. Hereafter for simplicity, we suppose $y_{m n}$ is the normalized value of criterion $n$ with respect to the inventory item $m$. Normalization of criteria has two main advantages. First, it avoids any problem arising from the criteria scale differences. Second, normalized data are fairly easy to interpret [32]. It is worthy to mention that there exist several normalization methods in the literature. Some of the well-known normalization methods are listed below [33].

(i) Ranking Method. It is the simplest normalization technique, which is not affected by outliers.

(ii) Standardization (or $z$-Scores) Method. It converts indicators to a common scale with a mean of zero and standard deviation of one.

(iii) Min-Max Method. It normalizes criteria to have an identical range $[0,1]$ by subtracting the minimum value and dividing by the range of the indicator values.

(iv) Distance to a Reference Method. It measures the relative position of a given indicator vis-à-vis a reference point.

The concerned problem is aggregating the performances of an inventory item in terms of different criteria into a single score (called aggregated performance or composite index) for the subsequent ABC classification. The R-model introduced by the Ramanathan [1] is as follows:

$$
\begin{aligned}
g I_{i}= & \max \sum_{n=1}^{N} w_{i n} y_{i n} \\
\text { s.t. } & \sum_{n=1}^{N} w_{i n} y_{m n} \leq 1 ; \quad m=1,2, \ldots, M, \\
& w_{\text {in }} \geq 0 ; \quad n=1,2, \ldots, N,
\end{aligned}
$$

where $g I_{i}$ for $i=1,2, \ldots, M$ indicates the aggregated performance of inventory item $i$ with respect to all of underlying criteria and $w_{\text {in }}$ is the weight of criterion $n$ which is generated endogenously by the R-model when evaluating item $i$. Objective function of the R-model is similar to the simple additive weighting (SAW) aggregation method, while the weights of criteria are changeable for each item and calculated endogenously by the model. Consequently, a set of indices $g I_{1}, g I_{2}, \ldots, g I_{M}$ for all items are provided by solving the R-model repeatedly for each inventory item. It should be noted that the R-model is very similar to an output maximizing multiplier DEA model with multiple outputs and one constant input [1]. It is noted that there are several weighting and aggregation methods in the literature. Some are taken from statistical models, such as factor analysis, data envelopment analysis, and unobserved components models (UCM), or from participatory methods like budget allocation processes (BAP), analytic hierarchy processes (AHP), and conjoint analysis (CA). Equal weighting (EW) is one of the simplest weighting methods. Furthermore, three simple but popular aggregation methods are the simple additive weighting (SAW) method, the weighted product (WP) method, and the weighted displaced ideal (WDI) method [33].

In spite of popularity of the R-model, Zhou and Fan [18] mathematically proved that if an item has a value dominating other items in terms of a certain criterion, it would always obtain an aggregated performance score of 1 even if it has severely bad values with respect to other criteria. This may lead to the situation where an item with a high value in an unimportant criterion but with low values in other important criteria is inappropriately classified as class A, which may not reflect the real position of this inventory item. Furthermore, the obtained weights provided by the R-model are often unrealistic and lead to poor discriminatory power among SKUs. That is, for each inventory item, the criteria which have good performance may receive extremely high weights and those having bad performance may receive extremely low weights leading to extreme weights that are often unrealistic and impractical because of ignoring the impact of criteria with extremely low weight values for ABC classification [31, 34]. Accordingly, this paper proposes a new MCIC model in which all inventory items are evaluated using a set of common weights to enable a fair comparison among them that differs with the R-model in which the inventory items are evaluated by different sets of weights. For doing so, let $d_{i}$ denote the performance score deviation of item $i$ from the unity when it is under evaluation. Now, by considering $g I_{i}=1-d_{i}$, the R-model can be reformulated as follows when evaluating item $i$ :

$$
\begin{array}{ll}
\min & d_{i} \\
\text { s.t. } & \sum_{n=1}^{N} w_{i n} y_{m n}+d_{m}=1 ; \quad m=1,2, \ldots, M \\
& w_{\text {in }} \geq 0, \quad d_{m} \geq 0 ; \quad n=1,2, \ldots, N, m=1,2, \ldots, M,
\end{array}
$$


where $d_{i}$ denotes the performance score deviation of item $i$, $0 \leq i \leq M$. Notably, two separate indices $i$ and $m$ are used in Model (2) that both of them are bounded as $0 \leq i, m \leq$ $M$. The first index is related to the $i$ th linear programming model that should be solved for measuring the score of item $i$. The first $M$ constraints of Model (2) indicate that when evaluating item $i$, the composite performance score of each item, that is, $\sum_{n=1}^{N} w_{i n} y_{m n}$ for $m=1,2, \ldots, M$, plus the related performance deviation from the unity must be equal to 1 .

It is worthwhile to point out when item $i$ is under evaluation, the resulting weight vector $\left(w_{i 1}, w_{i 2}, \ldots, w_{i N}\right)$ is similar for both Models (1) and (2) that provides the most favorable weights for this item. Also, $g I_{i}$ provided by Model (1) is equal to $1-d_{i}$ that is provided by Model (2). Notably, Model (2) should also be solved $M$ times to minimize the performance score deviation of each item. However, solving this model for each item separately provides totally $M$ different weight vectors for criteria, each of which might be unrealistic as mentioned before.

To overcome this deficiency, we propose a common weight DEA-like model as follows. The objective function of Model (2) only involves the performance score deviation of item $i$. However, to maximize the aggregated performance scores of all inventory items simultaneously as it is needed in a common weight framework, we need to reformulate this model via a set of common weights. For doing so, this paper uses the minimax approach to minimize the maximum efficiency deviation among all items. It is noteworthy to mention that the minimax efficiency score is employed to avoid yielding unrealistic weight dispersion and improve the discriminating power in the context of DEA models [35]. In this manner, the proposed common weight linear model can be written as follows:

$$
\begin{array}{ll}
\min & \eta \\
\text { s.t. } & \eta-d_{m} \geq 0 ; \quad m=1,2, \ldots, M, \\
& \sum_{n=1}^{N} w_{n} y_{m n}+d_{m}=1 ; \quad m=1,2, \ldots, M, \\
& w_{n} \geq 0, \quad d_{m} \geq 0 ; \quad n=1,2, \ldots, N, m=1,2, \ldots, M,
\end{array}
$$

where $w_{n}$ denotes the common weight with respect to criterion $n$ among all inventory items which is endogenously generated by solving Model (3). Notably, the constraints $\eta-$ $d_{m} \geq 0 ; \forall m$ assure that $\eta$ is the maximum of $d_{m}$ values while they do not change the feasible region of decision variables as discussed in Li and Reeves [35]. When Model (3) is solved, the composite index of all inventory items can be calculated by $1-d_{m}$ for $m=1,2, \ldots, M$. After determining the item scores, we sort them in a nonincreasing order and then classify them by the Pareto rule based principle of $\mathrm{ABC}$ analysis. The classifying distribution of inventory items performs based on their scores. For example, the classifying distribution, that is, $20 \%$ of the inventory items with best scores in class A, $30 \%$ in class $\mathrm{B}$, and $50 \%$ in class $\mathrm{C}$, can be considered.
According to the literature of common weights DEA models [31,34], all inventory items are concurrently evaluated using a set of common weights to enable a fair comparison among them which highly differs with the R-model in which each inventory item is evaluated by its own favored weights. Furthermore, the common weight structure of the proposed method improves the discriminatory power among all inventory items. Notably, unrealistic weight distribution is a main difficulty of some DEA models where some inputoutput weights achieve the extreme or zero values which in turn leads to wrong assessment of some DMUs as efficient units. However, as discussed by $\mathrm{Li}$ and Reeves [35] and Bal et al. [36], the minimax approach provides realistic input-output weights and improves the discrimination power among DMUs. Accordingly, the main reasons for applying the minimax approach to derive the final scores of inventory items are as follows.

The minimax approach does not lead to the most favorable weights for each inventory item under evaluation as the R-model does. Therefore, the items' scores generated by this approach are tighter than those provided by the Rmodel. In particular, if inventory item $i$ achieves score of 1 by employing the minimax approach, it must also achieve score of 1 by employing the R-model, since the minimax score requires $d_{i}=0$. However, if inventory item $i$ achieves score of 1 by employing the R-model, it may receive a score less than 1 by applying the minimax approach, because $g I_{i}=1$ or $d_{i}=0$ does not necessarily means that $\eta$ is being minimized. According to this discussion, it can be affirmed that the minimax approach generally results in fewer efficient items with score of 1 leading to discrimination power improvement of Model (3). Moreover, since $\eta$ is defined as a function of all deviation variables and each deviation variable is associated with a constraint, therefore, minimizing $\eta$ is equivalent to imposing rigorous constraints on common weight variables. In this way, the range of criteria weights is effectively restricted leading to moderating the homogeneity of weights dispersion on a common base and hence Model (3) provides a realistic and reasonable set of weights for the $\mathrm{ABC}$ analysis.

In spite of above descriptive advantages of proposed Model (3), its attractiveness can be more declared through clarifying its interesting mathematical properties as follows.

\section{(a) Model (3) Is always Feasible}

Proof. Suppose that all required data (i.e., the $y_{m n}$ values) in Model (3) are first normalized. So, we would have $0 \leq y_{m n} \leq$ $1, \forall m, n$. Then, the following solution is feasible for Model (3).

Consider $w_{n}=0$ for $n \neq k$ and $w_{k}=1 \Rightarrow d_{m}=1-y_{m k}$; $\forall m \Rightarrow \eta=\max _{m}\left\{1-y_{m k}\right\}$.

(b) $0 \leq d_{m} \leq 1, \forall m$

Proof. Taking into account the nonnegativity of the variables $w_{n}$ and $d_{m}$ for each $m$ and $n$ and the fact that $0 \leq y_{m n} \leq 1$, $\forall m, n$, it would be clear that $\sum_{n=1}^{N} w_{n} y_{m n} \geq 0, \forall m$. Therefore, according to the constraints $\sum_{n=1}^{N} w_{n} y_{m n}+d_{m}=1, \forall m$ we can conclude that $d_{m} \leq 1, \forall m$. 
(c) The Common Weights Generated by Proposed Model (3) Do Not Exceed 1 (i.e., $w_{n} \leq 1, \forall n$ )

Proof. Assume that for at least criterion $n$ for which we have $y_{\max }^{n}=\max _{m}\left\{y_{m n}\right\}=1, \operatorname{Model}(3)$ yields $w_{n}>1$. Then, the constraint $\sum_{n=1}^{N} w_{n} y_{m n}+d_{m}=1$ enforces the corresponding deviation variable, that is, $d_{m}$ must be negative, a contradiction. Therefore, the common weights generated by proposed Model (3) do not exceed 1 and we have $w_{n} \leq 1$, $\forall n$.

(d) Regarding Proposed Model (3). If ith item be minimax efficient, that is, $d_{i}=0$, then it is recognized as an efficient item by the R-model as well; that is, $g I_{i}=1$. However, if the $i$ th item be efficient by the R-model, it may be minimax inefficient by employing Model (3), since it may not be assured that $\eta$ is being minimized. Also, it can be concluded that if $g I_{i}<1$ by using Model (1), then Model (3) yields $d_{i}>0$ and consequently it will be inefficient since $1-d_{i}<1$. On the other hand, if a specific item achieves a score smaller than 1 in terms of Model (1), then it also gives a score less than 1 when applying Model (3).

\section{Numerical Example}

In this section, we provide a numerical example to illustrate the advantages of the proposed approach. The example presented in Table 1 is part of the data set used as a case study in the literature $[1,5,11,18,20,22]$. More details about this data set are reported in the next section. The data set contains 7 inventory items with three benefit-type criteria, that is, average unit cost (AUC), annual dollar usage (ADU), and lead time (LT). After normalization, Models (1) to (3) are applied and related results are given in Table 2.

To achieve inventory item scores, that is, $g I_{1}, g I_{2}, \ldots, g I_{7}$, Model (1) is separately solved for each inventory item by changing the objective function accordingly. In a similar way, Model (2) is repeatedly solved for each inventory item. For example, when evaluating inventory item 44, Models (1) and (2) can be written as follows.

Model (1). Consider

$$
g I_{4}=\max 1 w_{41}+0.426 w_{42}+0.2 w_{43}
$$

$$
\text { subject to }\left\{\begin{array}{l}
0.285 w_{41}+1 w_{42}+0 w_{43} \leq 1 \\
0.734 w_{41}+0.929 w_{42}+0 w_{43} \leq 1 \\
0.538 w_{41}+0.639 w_{42}+0.6 w_{43} \leq 1 \\
1 w_{41}+0.426 w_{42}+0.2 w_{43} \leq 1 \\
0.651 w_{41}+0.168 w_{42}+1 w_{43} \leq 1 \\
0.511 w_{41}+0.064 w_{42}+0.2 w_{43} \leq 1 \\
0 w_{41}+0 w_{42}+0.6 w_{43} \leq 1 \\
w_{41}, w_{42}, w_{43} \geq 0
\end{array}\right.
$$

TABle 1: Numerical example data.

\begin{tabular}{ccccc}
\hline$m$ & Item number & $\begin{array}{c}\text { Average unit } \\
\text { cost (\$) }\end{array}$ & $\begin{array}{c}\text { Annual dollar } \\
\text { usage (\$) }\end{array}$ & $\begin{array}{c}\text { Lead time } \\
\text { (week) }\end{array}$ \\
\hline 1 & Item S41 & 19.8 & 79.2 & 2 \\
2 & Item S42 & 37.7 & 75.4 & 2 \\
3 & Item S43 & 29.89 & 59.78 & 5 \\
4 & Item S44 & 48.3 & 48.3 & 3 \\
5 & Item S45 & 34.4 & 34.4 & 7 \\
6 & Item S46 & 28.8 & 28.8 & 3 \\
7 & Item S47 & 8.46 & 25.38 & 5 \\
\hline
\end{tabular}

Model (2). Consider

$\min d_{4}$

$$
\text { subject to }\left\{\begin{array}{l}
0.285 w_{41}+1 w_{42}+0 w_{43}+d_{1}=1, \\
0.734 w_{41}+0.929 w_{42}+0 w_{43}+d_{2}=1, \\
0.538 w_{41}+0.639 w_{42}+0.6 w_{43}+d_{3}=1, \\
1 w_{41}+0.426 w_{42}+0.2 w_{43}+d_{4}=1, \\
0.651 w_{41}+0.168 w_{42}+1 w_{43}+d_{5}=1, \\
0.511 w_{41}+0.064 w_{42}+0.2 w_{43}+d_{6}=1, \\
0 w_{41}+0 w_{42}+0.6 w_{43}+d_{7}=1, \\
w_{41}, w_{42}, w_{43} \geq 0, \quad d_{1}, d_{2}, \ldots, d_{7} \geq 0,
\end{array}\right.
$$

where $w_{41}, w_{42}$, and $w_{43}$ denote the weights of AUC, ADU, and LT criteria, respectively. After solving the above Models (1) and (2), the respective weight vectors $(1,0,0)$ and $(0.6747,0.543,0.4696)$ are found, respectively. Notably, item 44 dominates all items in terms of AUC and hence Model (1) generates high weight value of 1 for this criterion and assigns for other criteria weights, which is clearly unrealistic because of ignoring the impact of ADU and LT criteria completely and at the same time assigning an extreme weight value for the AUC. The obtained score for this item is 1 that is calculated as $g I_{4}$ by Model (1) and $\left(1-d_{4}\right)$ by Model (2). It is worth pointing out that the solution generated by using Models (1) and (2) for evaluating this item is not unique. It is actually software dependent. For example, the solutions of $\left(w_{41}, w_{42}, w_{43}, g I_{4}\right)=(1,0,0,1)$ and $\left(w_{41}, w_{42}, w_{43}, g I_{4}\right)=$ $(0.6747,0.543,0.4696,1)$ are optimal for Model (1) when evaluating item 44. According to the results of Table 2, seven different weight vectors are produced by employing Models (1) or (2) to calculate final scores of all items. However, these items can be more fairly and confidently evaluated by just a unique set of common weights by solving proposed Model (3) that is written as follows. 
TABLE 2: The results.

\begin{tabular}{lccccccccc}
\hline & & \multicolumn{2}{c}{ Model (1) } & \multicolumn{2}{c}{ Model (2) } & \multicolumn{2}{c}{ Model (3) } \\
& Item score & $w_{i 1}$ & $w_{i 2}$ & $w_{i 3}$ & Item score & $w_{i 1}$ & $w_{i 2}$ & $w_{i 3}$ & Item score \\
\hline Item 41 & 1 & 0 & 1 & 0 & 1 & 0 & 1 & 0 \\
Item 42 & 1 & 0.1504 & 0.9572 & 0 & 1 & 0.1504 & 0.9572 & 0 & 0.38861 \\
Item 43 & 1 & 0 & 1 & 0.6014 & 1 & 0 & 1 & 0.6014 & 0.80642 \\
Item 44 & 1 & 1 & 0 & 0 & 1 & 0.6747 & 0.543 & 0.4696 & 0.71328 \\
Item 45 & 1 & 0 & 0 & 1 & 1 & 0 & 0.7426 & 0.8755 \\
Item 46 & 0.5498 & 0.9198 & 0 & 0.4011 & 0.5498 & 0.9198 & 0 & 0.4011 & 0.38861 \\
Item 47 & 0.6 & 0 & 0 & 1 & 0.6 & 0 & 0 & 1 \\
\hline
\end{tabular}

Model (3). Consider

$\min \eta$

$$
\text { subject to }\left\{\begin{array}{l}
\eta-d_{m} \geq 0, \quad m=1,2, \ldots, 7, \\
0.285 w_{1}+1 w_{2}+0 w_{3}+d_{1}=1, \\
0.734 w_{1}+0.929 w_{2}+0 w_{3}+d_{2}=1, \\
0.538 w_{1}+0.639 w_{2}+0.6 w_{3}+d_{3}=1, \\
1 w_{1}+0.426 w_{2}+0.2 w_{3}+d_{4}=1, \\
0.651 w_{1}+0.168 w_{2}+1 w_{3}+d_{5}=1, \\
0.511 w_{1}+0.064 w_{2}+0.2 w_{3}+d_{6}=1, \\
0 w_{1}+0 w_{2}+0.6 w_{3}+d_{7}=1, \\
w_{1}, w_{2}, w_{3} \geq 0, \quad d_{1}, d_{2}, \ldots, d_{7} \geq 0,
\end{array}\right.
$$

where $w_{1}, w_{2}$, and $w_{3}$ denote the common weights of AUC, ADU, and LT, respectively. Proposed Model (3) provides the score of all items by a more distributed weight vector $(0.4759,0.2531,0.6477)$ that is both unique and more reasonable than those generated by Models (1) and (2). Furthermore, when comparing the items' score columns presented in Table 2, it can be affirmed that Model (3) gives more discriminated scores than those obtained by Models (1) and (2).

\section{Case Study}

The proposed common weight linear optimization model is applied for the same multicriteria inventory classification problem as discussed in the literature $[1,5,11,18,20,22]$. The data include 47 inventory items (SKUs) with four criteria, namely, annual dollar usage (ADU), average unit cost (AUC), critical factor (CF), and lead time (LT). However, we follow $\mathrm{Ng}$ [5], Zhou and Fan [18], Chen [20], and Hadi-Vencheh [22] studies and consider just ADU, AUC, and LT criteria for ABC classification, since $\mathrm{CF}$ is a categorical and discontinuous criterion and not suitable for linear models. Note that all the three criteria that are presented in Table 3 are positively related to the importance level of inventory items. The same classifying distribution, that is, 10 class A, 14 class B, and 23 class $\mathrm{C}$, is adopted for illustration and comparison purposes as considered in the earlier similar works.
Before applying our proposed model, all measures are normalized. Proposed Model (3) generates the common weights $0.2290,0.5474$, and 0.3453 for ADU, AUC, and LT criteria, respectively. The fifth column of Table 3 shows the optimal aggregated performance scores of inventory items by solving proposed Model (3). The ABC classification with the above three criteria and by using the proposed model, the Chen [20] model, the R-model, the ZF-model, the NG-model, and the HV-model [22] has also been listed in Table 3. As our proposed model and the Chen-model [20] and ZF-model are various extensions of the R-model, therefore, our quantitative comparisons are considerably focused on the results of these models.

Zhou and Fan [18] discuss that the ZF model which is an extended version of the R-model provides a more reasonable and encompassing index for ABC classification. When our model is compared to the ZF-model, 4 out of 47 inventory items do not have the same classification. For class A items identified by our proposed model, nine items are classified as group A items in both models. Also, 12 out of 14 class B items and 22 out of 23 class $C$ items are matched in both models. For instance, item S34 is classified as class A by the R-model as it has the highest lead time and this is the only criterion taken into account in the model. However, it is classified as class $\mathrm{B}$ in both our model and the ZF model since, in addition to the high lead time, they also take into consideration its low average unit cost.

When comparing our proposed model with the Chenmodel [20], 10 out of 47 items have different classifications. Items 18 and 45 are classified as class B by the Chen-model [20], while they are moved up to class A by applying Model (3). Two class A items and three class C items resulting from the Chen-model [20] exchanged their classifications and shifted to class B when applying proposed Model (3). Furthermore, three class B items sorted by the Chen-model [20] are moved down to class $\mathrm{C}$ by using the proposed approach. In particular, item 15 with the normalized scores of 0.323 (AUC), 0.143 (ADU), and 0.333 (LT) dominates item 44 with the normalized scores of 0.211 (AUC), 0.003 (ADU), and 0.333 (LT). Consequently, as item 44 is rated as class $\mathrm{C}$ by all methods, it is more appropriate that item 15 is shifted to a better class like $B$ which coincides with the results of proposed Model (3) and the Chen-model [20]. As another comparison, item 9 has the normalized data as 0.333 (AUC), 0.412 (ADU), and 0.833 (LT), which is classified as class A by applying all 


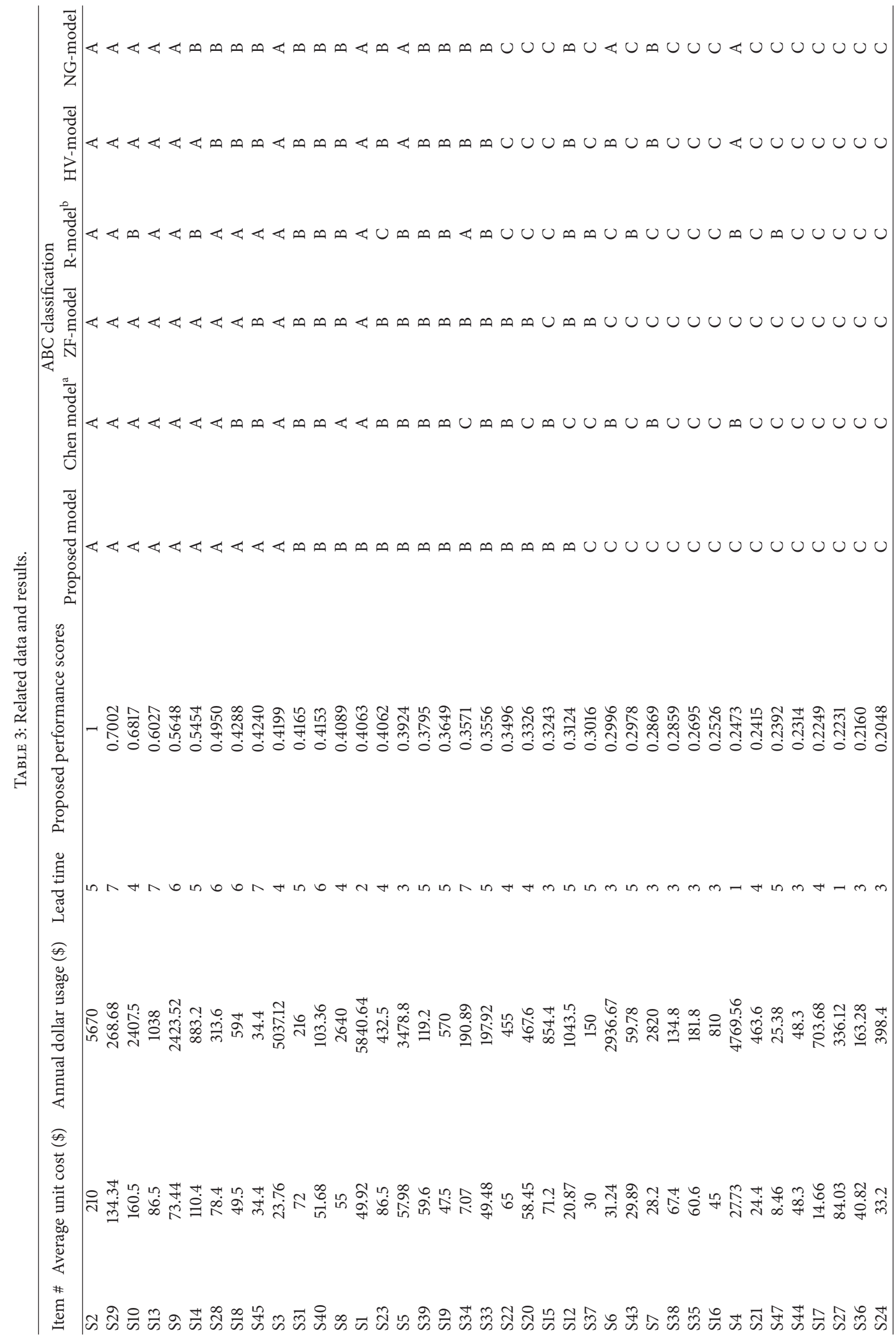




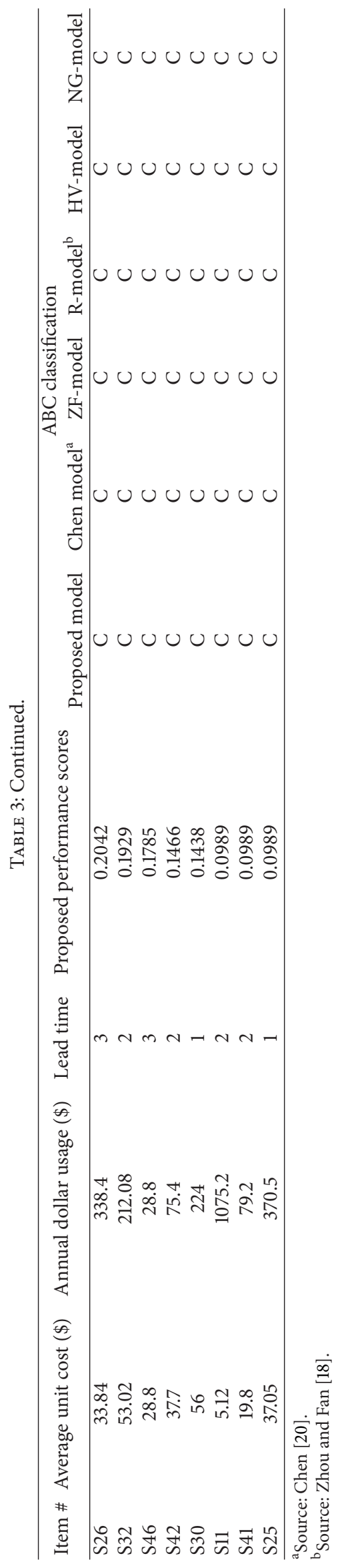


TABLE 4: Qualitative comparisons.

\begin{tabular}{lcccccc}
\hline Qualitative attributes & $\begin{array}{c}\text { Proposed } \\
\text { model }\end{array}$ & $\begin{array}{c}\text { Chen-model } \\
{[20]}\end{array}$ & $\begin{array}{c}\text { ZF-model } \\
{[18]}\end{array}$ & R-model [1] & $\begin{array}{c}\text { HV-model } \\
{[22]}\end{array}$ & $\begin{array}{c}\text { NG-model } \\
{[5]}\end{array}$ \\
\hline Type of optimization model & Linear & Linear & Linear & Linear & Nonlinear & - \\
Type of weights for evaluating each item & common & changeable & changeable & changeable & changeable & Independent \\
Number of models which must be solved & 1 & At least 2M & $2 \mathrm{M}$ & M & M & - \\
Requiring subjective information & No & No & Yes & No & Yes & Yes \\
\hline
\end{tabular}

models, and the normalized data of item 8 are as 0.243 (AUC), 0.445 (ADU), and 0.5 (LT). Item 9 dominates item 8 in terms of the AUC and LT criteria. Also, both items are relatively similar in terms of the ADU. Therefore, it is expected that item 8 might be inferior when compared to item 9 . Therefore, it is more appropriate that item 8 be moved down to a second-rate class like B which coincides with classification of Model (3) as well as four other models presented in Table 3. However, item 8 is rated as class A when employing the Chen-model [20].

According to the results, the classification results reported by the Chen-model [20] are different for 3 out of 47 items, that is, items 8,34 , and 12 , from other classifications while in the proposed classification, just item 1 has different classification from those provided by the other models. The reasons for this difference can be justified as follows. Consider the normalized data, $\{0.292$ (AUC), 0.074(ADU), 0.5(LT) , for item 22 which is classified as class B by the ZFmodel, the Chen-model [20], and our proposed model, and $\{0.219$ (AUC), 1 (ADU), 0.167(LT) $\}$ for item 1 which is evaluated as class A by five models presented in Table 3. It is clear that item 22 dominates item 1 in terms of AUC and LT criteria. Furthermore, proposed Model (3) assigns larger values for the weights of AUC and LT than the weight of ADU. In this way, item 22 is considerably superior to item 1 and hence it is more appropriate to put item 1 in an inferior class like class $\mathrm{B}$ which matches with our proposed classification. However, the main difference between ours and other models is that our composite index is calculated based on a unique set of common weights among all items.

When the proposed model is compared with the HVmodel [22] that is an extended version of the NG-model, 11 out of 47 inventory items are not coincident. Among the class A items recognized in our proposed model, 7 out of 10 items are classified as class A in both models. Furthermore, 9 out of 14 class B items and 20 out of 23 class C items are similarly classified in both models.

\section{Discussion}

To alleviate deficiencies of the R-model, Zhou and Fan [18] proposed another DEA-based model that aggregates the best and worst performance scores of each item using a control parameter called $\lambda$ to obtain the ZF-index for ABC classification. The poor discrimination power in the R-model is also improved in the ZF-model. However, choosing an appropriate value for this subjective parameter is crucial and depends on the decision maker's preference on two extreme cases. This flexibility may also sometimes cause difficulty for decision makers to make a subjective choice in specifying the value of $\lambda$. Furthermore, different values of $\lambda$ may lead to distinct, misleading, and nonunique results. It is noteworthy to mention that $2 M$ linear optimization models must be solved in order to calculate the ZF-index for all SKUs. Furthermore, Chen [20] proposed an alternative model for $\mathrm{ABC}$ analysis whose structure is similar to the ZF-model but without any subjectivity.

$\mathrm{Ng}$ [5] proposes a novel and easy-to-use method that does not require a linear optimizer for $\mathrm{ABC}$ analysis. Besides its many advantages, Hadi-Vencheh [22] discusses that the Ngmodel leads to a situation where the NG-index for each item is independent from the obtained weights. Consequently, Hadi-Vencheh [22] improves the NG-model and constructs a nonlinear programming model that keeps the impacts of weights when calculating the final indices. The HV-model is solved for each inventory item repeatedly (i.e., it requires to run $M$ nonlinear programming models) and a different set of weights is obtained for calculating the final index of each item. Moreover, both the NG and HV models need prior assumption on the importance order of criteria which is determined subjectively by the decision maker. It should be noted that when the number of criteria is large, it is an overwhelming task for the decision maker to rank all criteria.

Relying on the above discussions, the proposed method generates a set of common weights for evaluating all items simultaneously that obviously improves the discrimination power among them. Also, the proposed method removes any subjectivity which may lead to confusing interpretation of $\mathrm{ABC}$ classification results and hence provides unique $\mathrm{ABC}$ classification results. Table 4 shows the several merits of our proposed method in summary when comparing it with the recently developed methods for $\mathrm{ABC}$ inventory classification.

As highlighted by Chen et al. [37], common weights strategy to DEA has several features. First, the common weights strategy diminishes computational complexity/time compared with the traditional model. Second, common weights models show higher discrimination power than other classical models. However, for a typical MCIC problem, the proposed common weights strategy incorporated in the minimax approach in this paper has the following merits.

(1) Despite other existing methods that generate several sets of weights for the criteria, the proposed method obtains a unique set of common weights for evaluating all inventory items which is very essential for a fair classification of items and provides unique results for $\mathrm{ABC}$ analysis.

(2) The proposed model like the models presented by Chen $[19,20]$ requires no subjective information 
leading to provide a unique classification results for inventory managers while some recent $\mathrm{MCIC}$ approaches do. Requiring any subjective information may lead to nonunique and misleading results for the $\mathrm{ABC}$ analysis, making difficulty in reaching a final decision.

(3) The proposed method is very efficient from the computational point of view since only a LP model needs to be solved while the other methods need that several linear or even nonlinear models to be solved.

(4) The common weight structure incorporated in our proposed method improves the poor discrimination power of other methods especially the R-model by providing a full-ranked vector of items based upon their composite performance scores.

Relying on the aforementioned merits, it can be concluded that the proposed model is an easy-to-understand and easy-to-use method that helps inventory managers and practitioners to manage inventory items in reality. The proposed method can be applied to industry. Furthermore, it is easy to apply for human managers. The only background required for implementing our proposed model is to know how to solve linear programming models via a commercial software like LINGO. In summary, inventory managers can perform the following steps to classify their inventory items.

(1) Provide normalized measures by the transformation procedure presented in the paper.

(2) Solve proposed Model (3) on normalized data by the commercial software.

(3) Provide item scores by formula $1-d_{i}$ for $i=1,2$, $\ldots, M$ and then sort them in a nonincreasing order.

(4) Classify inventory items by the Pareto rule based principle of $\mathrm{ABC}$ analysis.

\section{Concluding Remarks}

This paper addresses the ABC inventory classification problem through the multiple criteria inventory classification (MCIC) approach and proposes a common weight linear optimization method that enables us to classify inventory items using a set of common weights in an efficient and effective way. The numerical results along with some qualitative assessments confirm the superiority of the proposed method when compared with the previously developed relevant techniques.

Recently, finding common weights based on the DM's preference information is addressed by Jahanshahloo et al. [38] in the DEA literature that measures the preferences of decision maker when generating the common weights. Accordingly, incorporating the inventory manager's preferences in a new model to derive common weights is proposed for future research in the context of MCIC. Furthermore, all above-mentioned DEA-based methods in this paper can only take into account quantitative criteria for dealing with MCIC problem.

\section{Conflict of Interests}

The authors declare that there is no conflict of interests regarding the publication of this paper.

\section{Acknowledgment}

The authors are grateful to the respected reviewers for their valuable comments in preparation of the revised paper.

\section{References}

[1] R. Ramanathan, "ABC inventory classification with multiplecriteria using weighted linear optimization," Computers and Operations Research, vol. 33, no. 3, pp. 695-700, 2006.

[2] C.-W. Chu, G.-S. Liang, and C.-T. Liao, "Controlling inventory by combining ABC analysis and fuzzy classification," Computers \& Industrial Engineering, vol. 55, no. 4, pp. 841-851, 2008.

[3] E. A. Silver, D. F. Pyke, and R. Peterson, Inventory Management and Production Planning and Scheduling, Wiley, New York, NY, USA, 1998.

[4] B. E. Flores and D. C. Whybark, "Implementing multiple criteria ABC analysis," Journal of Operations Management, vol. 7, no. 1-2, pp. 79-85, 1987.

[5] W. L. Ng, "A simple classifier for multiple criteria ABC analysis," European Journal of Operational Research, vol. 177, no. 1, pp. 344-353, 2007.

[6] H. A. Guvenir and E. Erel, "Multicriteria inventory classification using a genetic algorithm," European Journal of Operational Research, vol. 105, no. 1, pp. 29-37, 1998.

[7] J. Huiskonen, "Maintenance spare parts logistics: special characteristics and strategic choices," International Journal of Production Economics, vol. 71, no. 1-3, pp. 125-133, 2001.

[8] F. Y. Partovi and M. Anandarajan, "Classifying inventory using an artificial neural network approach," Computers and Industrial Engineering, vol. 41, no. 4, pp. 389-404, 2001.

[9] A. Bhattacharya, B. Sarkar, and S. K. Mukherjee, "Distancebased consensus method for ABC analysis," International Journal of Production Research, vol. 45, no. 15, pp. 3405-3420, 2007.

[10] J. Rezaei and S. Dowlatshahi, "A rule-based multi-criteria approach to inventory classification," International Journal of Production Research, vol. 48, no. 23, pp. 7107-7126, 2010.

[11] B. E. Flores, D. L. Olson, and V. K. Dorai, "Management of multicriteria inventory classification," Mathematical and Computer Modelling, vol. 16, no. 12, pp. 71-82, 1992.

[12] F. Y. Partovi and J. Burton, "Using the analytic hierarchy process for ABC analysis," International Journal of Operations \& Production Management, vol. 13, no. 9, pp. 29-44, 1993.

[13] A. Hadi-Vencheh and A. Mohamadghasemi, "A fuzzy AHPDEA approach for multiple criteria $\mathrm{ABC}$ inventory classification," Expert Systems with Applications, vol. 38, no. 4, pp. 33463352, 2011.

[14] Y. Chen, K. W. Li, D. Marc Kilgour, and K. W. Hipel, "A casebased distance model for multiple criteria ABC analysis," Computers and Operations Research, vol. 35, no. 3, pp. 776-796, 2008.

[15] Y.-Y. Xiao, R.-Q. Zhang, and I. Kaku, "A new approach of inventory classification based on loss profit," Expert Systems with Applications, vol. 38, no. 8, pp. 9382-9391, 2011. 
[16] C.-Y. Tsai and S.-W. Yeh, "A multiple objective particle swarm optimization approach for inventory classification," International Journal of Production Economics, vol. 114, no. 2, pp. 656666, 2008.

[17] M.-C. Yu, "Multi-criteria ABC analysis using artificial-intelligence-based classification techniques," Expert Systems with Applications, vol. 38, no. 4, pp. 3416-3421, 2011.

[18] P. Zhou and L. Fan, "A note on multi-criteria ABC inventory classification using weighted linear optimization," European Journal of Operational Research, vol. 182, no. 3, pp. 1488-1491, 2007.

[19] J.-X. Chen, "Peer-estimation for multiple criteria ABC inventory classification," Computers \& Operations Research, vol. 38, no. 12, pp. 1784-1791, 2011.

[20] J.-X. Chen, "Multiple criteria ABC inventory classification using two virtual items," International Journal of Production Research, vol. 50, no. 6, pp. 1702-1713, 2012.

[21] R. E. Stanford and W. Martin, “Towards a normative model for inventory cost management in a generalized ABC classification system," Journal of the Operational Research Society, vol. 58, no. 7, pp. 922-928, 2007.

[22] A. Hadi-Vencheh, "An improvement to multiple criteria ABC inventory classification," European Journal of Operational Research, vol. 201, no. 3, pp. 962-965, 2010.

[23] R. H. Teunter, M. Z. Babai, and A. A. Syntetos, "ABC Classification: service levels and inventory costs," Production and Operations Management, vol. 19, no. 3, pp. 343-352, 2010.

[24] J. Park, H. Bae, and J. Bae, "Cross-evaluation-based weighted linear optimization for multi-criteria $\mathrm{ABC}$ inventory classification," Computers \& Industrial Engineering, vol. 76, pp. 40-48, 2014.

[25] B. Soylu and B. Akyol, "Multi-criteria inventory classification with reference items," Computers and Industrial Engineering, vol. 69, no. 1, pp. 12-20, 2014.

[26] A. Bacchetti, F. Plebani, N. Saccani, and A. A. Syntetos, "Empirically-driven hierarchical classification of stock keeping units," International Journal of Production Economics, vol. 143, no. 2, pp. 263-274, 2013.

[27] J. Rezaei and N. Salimi, "Optimal ABC inventory classification using interval programming," International Journal of Systems Science, 2013.

[28] S. A. Torabi, S. M. Hatefi, and B. Saleck Pay, "ABC inventory classification in the presence of both quantitative and qualitative criteria," Computers and Industrial Engineering, vol. 63, no. 2, pp. 530-537, 2012.

[29] S. M. Hatefi, S. A. Torabi, and P. Bagheri, "Multi-criteria ABC inventory classification with mixed quantitative and qualitative criteria," International Journal of Production Research, vol. 52, no. 3, pp. 776-786, 2014.

[30] F. Lolli, A. Ishizaka, and R. Gamberini, "New AHP-based approaches for multi-criteria inventory classification," International Journal of Production Economics, vol. 156, pp. 62-74, 2014.

[31] E. E. Karsak and S. S. Ahiska, "A common-weight MCDM framework for decision problems with multiple inputs and outputs," in Computational Science and Its Applications-ICCSA 2007, vol. 4705 of Lecture Notes in Computer Science, pp. 779790, Springer, Berlin, Germany, 2007.

[32] P. Zhou, B. W. Ang, and K. L. Poh, "Comparing aggregating methods for constructing the composite environmental index: an objective measure," Ecological Economics, vol. 59, no. 3, pp. 305-311, 2006.
[33] M. Nardo, M. Saisana, A. Saltelli, S. Tarantola, A. Hoffman, and E. Giovannini, "Handbook on constructing composite indicators: methodology and user guide," OECD Statistics Working Paper 2005/3, 2005.

[34] E. E. Karsak and S. S. Ahiska, "Practical common weight multicriteria decision-making approach with an improved discriminating power for technology selection," International Journal of Production Research, vol. 43, no. 8, pp. 1537-1554, 2005.

[35] X.-B. Li and G. R. Reeves, "Multiple criteria approach to data envelopment analysis," European Journal of Operational Research, vol. 115, no. 3, pp. 507-517, 1999.

[36] H. Bal, H. H. Örkcü, and S. Çelebioğlu, "Improving the discrimination power and weights dispersion in the data envelopment analysis," Computers and Operations Research, vol. 37, no. 1, pp. 99-107, 2010.

[37] Y.-W. Chen, M. Larbani, and Y.-P. Chang, "Multiobjective data envelopment analysis," Journal of the Operational Research Society, vol. 60, no. 11, pp. 1556-1566, 2009.

[38] G. R. Jahanshahloo, M. Zohrehbandian, A. Alinezhad, S. A. Naghneh, H. Abbasian, and R. K. Mavi, "Finding common weights based on the DM's preference information," Journal of the Operational Research Society, vol. 62, no. 10, pp. 1796-1800, 2011. 


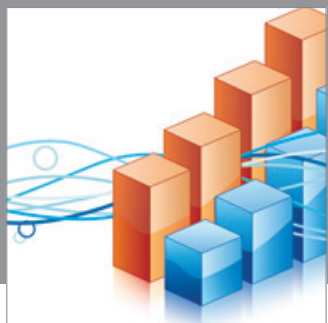

Advances in

Operations Research

mansans

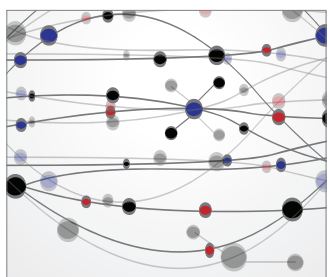

The Scientific World Journal
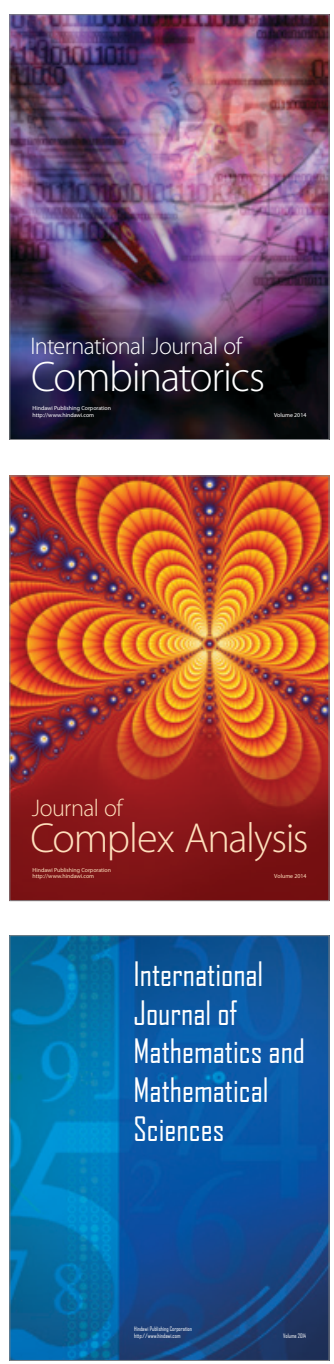
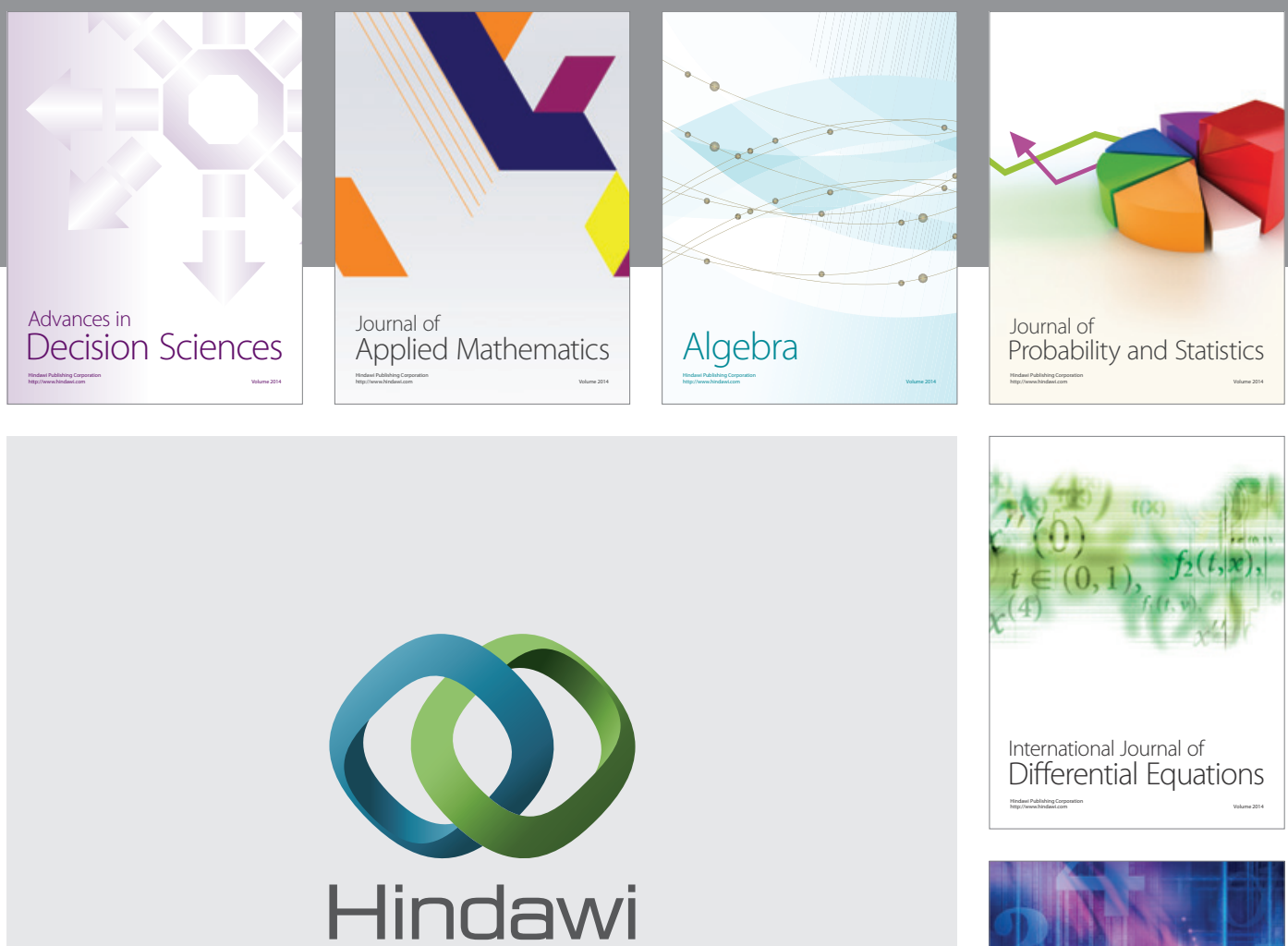

Submit your manuscripts at http://www.hindawi.com
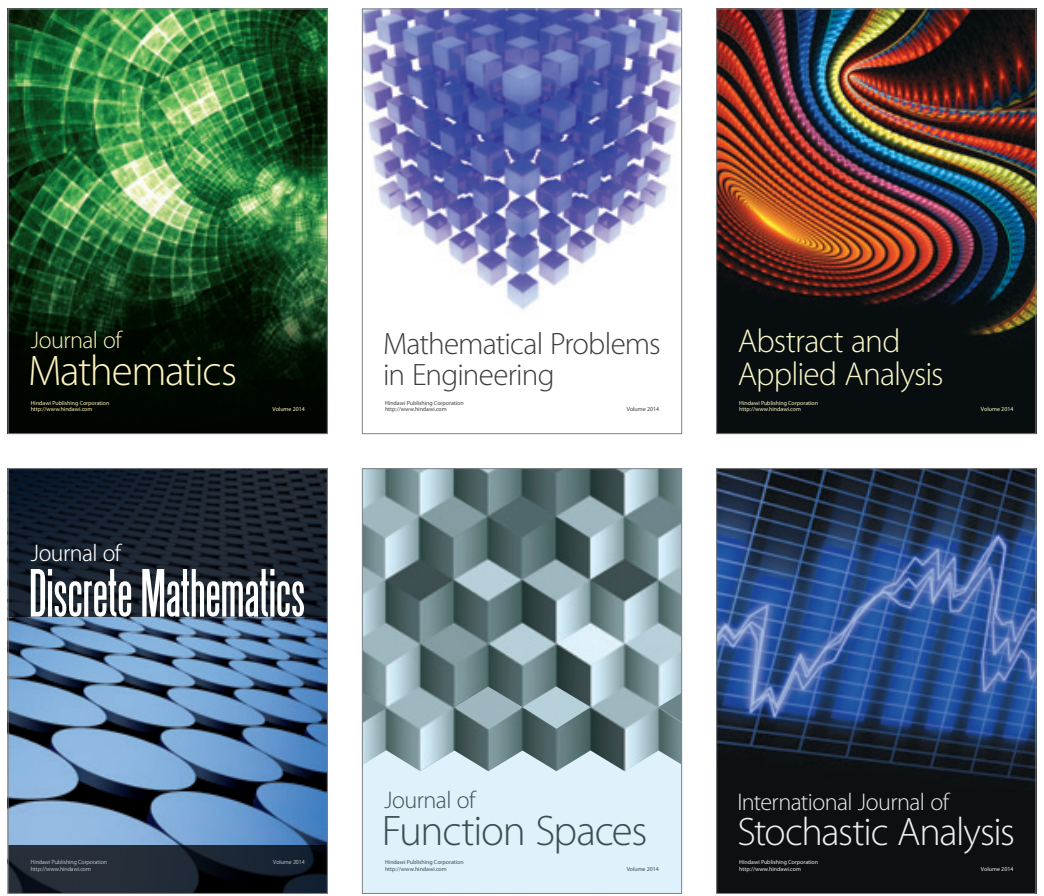

Journal of

Function Spaces

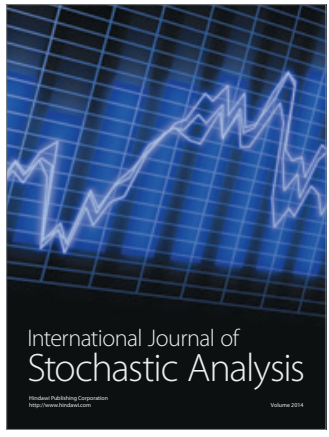

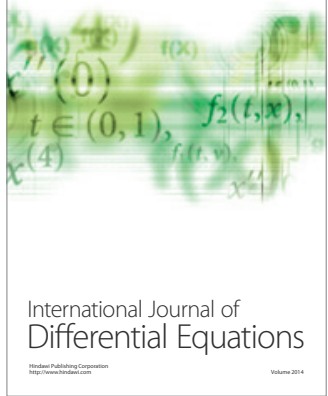
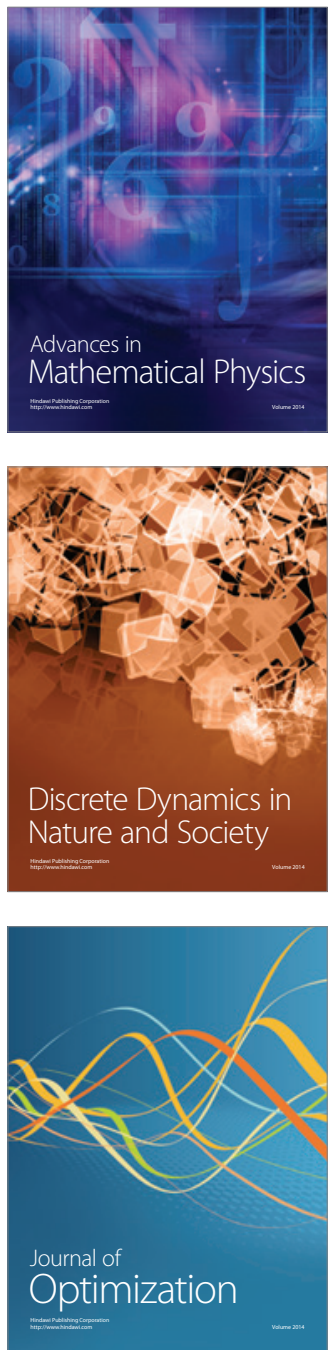